Article

\title{
X-ray Shaping of Planetary Nebulae
}

\author{
Martín A. Guerrero (D) \\ Instituto de Astrofísica de Andalucía, IAA-CSIC, 18080 Granada, Spain; mar@iaa.es \\ Received: 30 July 2018; Accepted: 4 September 2018; Published: 11 September 2018
}

Abstract: The stellar winds of the central stars of planetary nebulae play an essential role in the shaping of planetary nebulae. In the interacting stellar winds model, the fast stellar wind injects energy and momentum, which are transferred to the nebular envelope through an X-ray-emitting hot bubble. Together with other physical processes, such as the ionization of the nebular envelope, the asymmetrical mass-loss in the asymptotic giant branch (AGB), and the action of collimated outflows and magnetic fields, the pressurized hot gas determines the expansion and evolution of planetary nebulae. Chandra and XMM-Newton have provided us with detailed information of this hot gas. Here in this talk I will review our current understanding of the effects of the fast stellar wind in the shaping and evolution of planetary nebulae and give some hints of the promising future of this research.

Keywords: planetary nebula; X-ray; stellar evolution

\section{Introduction}

Planetary nebulae (PNe) are the descendants of low- to intermediate-mass $\left(0.8-1.0 M_{\odot} \leq M_{i} \leq\right.$ 8-10 $M_{\odot}$ ) stars. They are formed when such stars climb up to the tip of the asymptotic giant branch (AGB) and heavy mass loss through slow $\left(\sim 10 \mathrm{~km} \cdot \mathrm{s}^{-1}\right)$ and dense winds powered by radiation pressure on neutral dust grains eject the stellar envelope. As the hot stellar core is exposed, the sudden increase in ionizing flux and fast stellar wind (1000-4000 $\left.\mathrm{km} \cdot \mathrm{s}^{-1}\right)$ [1] developed by the central star will ionize and sweep up the slow AGB wind to form a PN.

In the now classical interacting stellar winds (ISW) model [2,3], the expansion of PNe is powered by isotropic fast stellar winds. As these stellar winds encounter the slow AGB wind, a reverse-shock heats the stellar wind up to X-ray-emitting million-Kelvin temperatures, resulting in an onion-like structure similar to that proposed for Wolf-Rayet bubbles by Weaver et al. [4]. The extreme heating of this shock produces an over-pressurized hot bubble that works like a spherical piston, displacing undisturbed gas upstream into into a thin dense rim. Here I review our current understanding of the hot gas in PNe and describe some of the advances that can be achieved by future X-ray observatories.

\section{Past and Current X-ray Observations of PNe}

Hot bubbles have an X-ray limb-brightened morphology, with diffuse X-ray emission confined within the central cavity defined by the inner rim of PNe, as revealed in the first Chandra observations of PNe [5,6]. Since these early observations, Chandra and XMM-Newton have produced exquisite images and spectra of the diffuse X-ray emission from the hot bubbles of PNe [7-14].

All these results have been put in context and expanded by the analysis and new deep X-ray observations obtained in the framework of the Chandra Planetary Nebula Survey (ChanPlaNS) [15-17], which has surveyed the X-ray emission from $\sim 50 \mathrm{PNe}$, all within $1.5 \mathrm{kpc}$, with a total exposure time over $1 \mathrm{Ms}$. These Chandra X-ray observations have made clear that the detections of diffuse X-ray emission is always confined within sharp closed innermost optical shells (i.e., the hot bubbles) $[15,16]$. So far, there is no evidence of X-ray emission associated with fast collimated outflows, but for the highly collimated ultrafast $\left(>1000 \mathrm{~km} \cdot \mathrm{s}^{-1}\right)$ proto-PN Hen 3-1475 [18]. 
The emerging picture implies that a closed inner shell is necessary but not sufficient for the detection of hot $\mathrm{X}$-ray-emitting gas. This is only present in young $\mathrm{PNe}$, as testified by the high electron density $\left(N_{e}>1000 \mathrm{~cm}^{-3}\right)$ and small nebular radius $(<0.15 \mathrm{pc})$ of PNe with diffuse X-ray emission [16]. Obviously, the rapidly changing stellar wind, whose mechanical power declines in a few thousand years, and the fast nebular expansion make the X-ray luminosity drop quickly [19]. As a general rule, diffuse X-ray emission is very unlikely among PNe with dynamical ages above 5000 years [14].

\section{The Future of X-ray Studies of PNe}

\subsection{Future X-ray Observations of PNe}

The current Chandra and XMM-Newton CCD spectroscopic observations of PNe have limited spectral resolution (typically $\approx 70 \mathrm{eV}$ ), which is degraded in cases of low count rate [20]. High-dispersion grating spectroscopic observations of the two X-ray brightest PNe, BD+30 3639 and NGC 6543, have provided interesting insights into the physical processes associated with the production of hot gas in PNe.

The Chandra X-ray Observatory's Low Energy Transmission Gratings and Advanced CCD Imaging Spectrometer (LETG/ACIS-S) were used to obtain a deep high-dispersion spectrum of BD+30 3639 [21]. The brightest lines in this spectrum are associated with highly ionized $(\mathrm{H}$ - and He-like) species of $\mathrm{C}$, $\mathrm{O}$, and Ne, such as O VIII $\lambda 18.97$, C VI $\lambda 33.6$, Ne IX $\lambda \lambda 13.45,13.55,13.7$, and O VII $\lambda \lambda 21.60,21.80,22.10$. The hot plasma has a range of temperatures between $1.7 \times 10^{6} \mathrm{~K}$ and $2.9 \times 10^{6} \mathrm{~K}$, with chemical abundances consistent with the enhancement of carbon and neon.

As for NGC 6543, a 435 ks XMM-Newton Reflection Grating Spectrometer (RGS) exposure provided only a clear detection of the He-like triplet of O VII at $22 \AA$ [22], although it must be noted that only $70 \mathrm{ks}$ were useful because high background conditions affected most of these observations. The absence of the H-like $\mathrm{O}$ VIII points to lower $T_{X}$ than in $\mathrm{BD}+30^{\circ} 3639, k T \approx 0.147 \mathrm{keV}$ (or $T_{X} \approx 1.7 \times 10^{6} \mathrm{~K}$ ). The N VII line at $24.78 \AA$ was tentatively detected. The weakness of the $\mathrm{N}$ lines implies a low N/O ratio, favoring nebular abundances and high mixing. The lack of $\mathrm{Ne}$ and $\mathrm{C}$ lines indicates lower $\mathrm{Ne} / \mathrm{O}$ and $\mathrm{C} / \mathrm{O}$ than in $\mathrm{BD}+30^{\circ} 3639$.

These high-dispersion grating $\mathrm{X}$-ray spectroscopic observations are at the technical limit of Chandra and XMM-Newton. All other X-ray-emitting PNe are fainter or have lower surface brightness than BD $+30^{\circ} 3639$ and NGC 6543 . We should not expect a breakthrough in our understanding of the production of hot gas in PNe and their effects in their expansion and evolution until the upcoming ATHENA, the Advanced Telescope for High-Energy Astrophysics. ATHENA is the second large-class ESA mission (L2), which is expected to be launched by 2028. ATHENA will have two main instruments, the Wide Field Imager (WFI) and the X-ray Integral Field Unit (XIFU). The former will have unprecedented sensitivity to diffuse $X$-ray emission, whereas the latter will be capable of providing high-dispersion spectra of sources as faint as PNe. This is illustrated in Figure 1. A single $10 \mathrm{ks}$ WFI exposure can very easily determine whether the abundances of the X-ray-emitting plasma in NGC 6543 are nebular or stellar. Furthermore, a 20 ks XIFU exposure can even look into the details of the plasma physics to accurately determine the physical conditions and chemical abundances. 

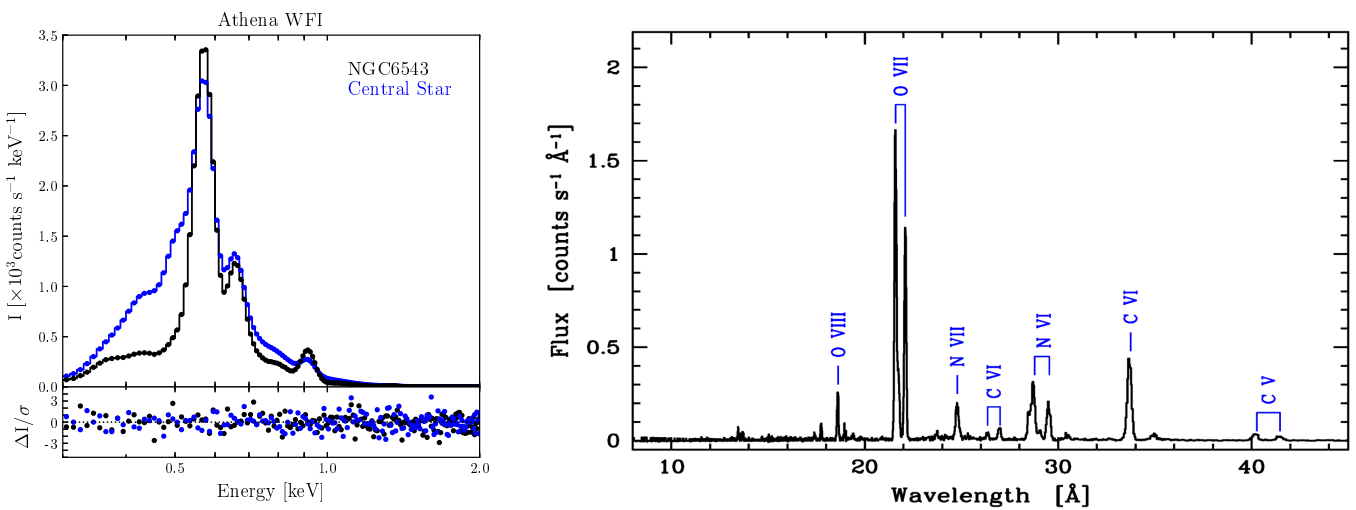

Figure 1. (left) Simulated ATHENA (Advanced Telescope for High-Energy Astrophysics) Wide Field Imager (WFI) 10 ks spectrum of NGC 6543 modeled using nebular (black) or stellar (blue) abundances. (right) Simulated ATHENA X-ray Integral Field Unit (XIFU) 20 ks spectrum of NGC 6543.

\subsection{Effects of the Stellar and Nebular Evolution}

The stellar evolution determines the mechanical luminosity of the stellar wind (i.e., the energy and momentum injected into the hot bubble). The nebular evolution, particularly the volume of the hot bubble, determines the energy density inside it. The most complete 1-D models accounting for the effects of the stellar and nebular evolution are those by Steffen et al. [19]. Their predictions compare reasonably well with detailed X-ray observations of PNe [14].

More complete 2-D models have the possibility to include effects of turbulent mixing. Previous models $[23,24]$ have been superseded by the recent 2-D hydro-dynamical simulations presented by Toalá \& Arthur [25]. These new models show the relevance of turbulent mixing and heat conduction on the evolution of the hot gas in PNe [20]. Rayleigh-Taylor instabilities at the interface are shown to be responsible for shadowing instabilities, which have notable effects in the mid-IR (dust) and near-IR $\mathrm{H}_{2}$ (molecular) morphologies [26]. Future multi-dimensional hydro-dynamical models will also need to account for faster and brighter CSPN evolutionary tracks [27].

The end of the pressure-dominated hot bubble occurs when the stellar wind mechanical power drops below a limit where it is no longer able to push the nebular rim. The rim thickness then increases and, at some moment in the late evolution, it would even be able to backfill the central cavity [28,29]. However, it is difficult to compare theoretical predictions of this effect with observations given the different distances (and spatial scales) of PNe, projection effects, and small-scale nebular features [30].

Alternatively, the hot bubble can get pinched. Then, it would be unable to retain the hot gas, as shown in most PNe with open bipolar lobes or pinched hot inner rims [7]. Similar processes have been investigated in Wolf-Rayet bubbles, given their large angular size. The nebula S 308 is one of these cases, where the highly pressurized hot gas is producing a blowout feature on the otherwise round nebular morphology [31,32].

\subsection{Effects of Asymmetries in the AGB Envelope or Fast Wind}

The effects of asymmetric mass loss on the hot bubble have received little attention up to now. The symmetric fast stellar wind can impinge on an asymmetrical distribution of the nebular envelope, due either to asymmetrical mass loss during the AGB or to a symmetric AGB wind shaped by collimated outflows [33].

If the fast stellar wind is much faster than the AGB wind, the hot bubble is expected to become isobaric and push away the asymmetric envelope. The shape of the PN becomes self-similar for stellar winds with constant (unrealistic) properties [34]. More detailed simulations show that the action of a symmetric fast stellar wind on an asymmetric AGB envelope may result in elliptical PNe, whereas spherical PNe are unlikely to evolve from bipolar proto-PNe or elliptical PNe [35]. 
Alternatively, the fast stellar wind might be collimated. The interaction of a fast collimated fast winds (CFW) with a spherical AGB wind has been investigated by Lee \& Sahai [36] and Akashi \& Soker [37]. A $1000 \mathrm{~km} \cdot \mathrm{s}^{-1} \mathrm{CFW}$ would be able to produce a bow-shock driven shell, where the wind itself would be surrounded by a hot X-ray-emitting cocoon.

\subsection{Effects of Magnetic Fields}

Heat conduction is largely influenced by magnetic fields, suppressing conduction normal to the field. Even a weak stellar magnetic field, $B_{\star} \leq 1 \mathrm{G}$, can result in asymmetric thermal conduction in colliding stellar winds, because the reduced mixing along the equatorial direction implies an additional pumping (more pressure) along the main axis [38,39].

However, these results do not account for radiative and photo-ionization effects, which tend to make isobaric the pressure inside the hot bubble. Magneto-radiative-hydrodynamical simulations are required to solve this issue.

\subsection{Ignored Physics}

The limitations of the current X-ray observations (and theoretical models) of hot bubble in PNe do not allow us to investigate a series of physical processes with potential implications on the evolution of PNe. For instance, the hot bubble is assumed to be isobaric. We have seen above that magnetic fields may change this situation, but the interaction of the hot bubble with the AGN envelope is certainly complex and can produce short-lived non-isobaric regions. The 2-D radiative hydro-dynamical simulations of Toalá \& Arthur [20] predict the presence of pockets of plasma with varying physical conditions at the interface between the hot bubble and the nebular rim. Slow-moving cold clumps may even provide pick-up ions, that is, suprathermal particles that may lower the post-shock velocity of the fast wind and reduce the temperature of the X-ray-emitting plasma in the hot bubble [40].

Charge-exchange reactions (CXE) may be important between ions of the stellar winds and the nebular envelope. These have been suggested to play an important role in the interactions of the post-born-again and present fast stellar winds with H-poor ejecta in the born-again PNe A 30 and A 78. In this particular case, soft thermal emission from $\mathrm{H}$-poor knots ablated by the stellar wind, which is mass-loaded to raise its density and damp its velocity, produces the extremely soft spectrum mostly consistent with a single C VI line emission [41,42]. The evaporation of clumps of cold material, which may survive inside the hot bubble and photo-evaporate $[43,44]$, may produce similar effects.

As important can be the recombination of carbon ions from the hot bubble with cool $\mathrm{e}^{-}$. These can cross the contact discontinuity into the cold nebula to produce the continuum emission excess attributed to recombination lines of C VI in $\mathrm{BD}+30^{\circ} 3639$ [45].

\section{Summary}

The hot gas resulting from the wind-wind interaction is one main PN shaping agent (in addition to collimated outflows, ionization, azimuthal density gradients, etc.) Hot gas mostly affects the early post-AGB evolution. Once the stellar wind power declines, the hot bubble pressure is not sufficient to maintain the nebular expansion. Rims depressurize very quickly, within 5000 years from the PN formation, or even faster if the hot bubble gets pinched.

Hot gas reveals complex physical processes: RRC, CEX, local non-equilibrium, weak magnetic fields, etc. Delicate interactions can produce subtle effects, such as shadowing instabilities, cold clumps evaporation, etc. The community needs to get ready for the next generation of X-ray telescopes, with all its observational and theoretical homework done. 
Funding: This research was funded by Ministerio de Economía, Industria y Competitividad, Gobierno de España, grant number: AYA2014-57280-P.

Acknowledgments: The author acknowledges the support of the grant AYA2014-57280-P, co-funded with FEDER funds. He also appreciates the invitation of the Asymmetrical Planetary Nebulae VII Scientific Organizing Committee for the invited talk, which is reported here.

Conflicts of Interest: The author declares no conflict of interest.

\section{References}

1. Guerrero, M.A.; De Marco, O. Analysis of far-UV data of central stars of planetary nebulae: Occurrence and variability of stellar winds. Astron. Astrophys. 2013, 553, A126. [CrossRef]

2. Kwok, S. Effects of stellar mass loss on the formation of planetary nebulae. In Planetary Nebulae; Proceedings of the Symposium; Dordrecht, D., Ed.; Reidel Publishing Co.: London, UK, 1983; pp. 293-302.

3. Frank, A.; Mellema, G. A radiation-gasdynamical method for numerical simulations of ionized nebulae: Radiation-gasdynamics of PNe I. Astron. Astrophys. 1994, 289, 937-945.

4. Weaver, R.; McCray, R.; Castor, J.; Shapiro, P.; Moore, R. Interstellar bubbles. II-Structure and evolution. Astrophys. J. Lett. 1977, 218, 377-395. [CrossRef]

5. Kastner, J.H.; Soker, N.; Vrtilek, S.D.; Dgani, R. Chandra X-ray Observatory Detection of Extended X-ray Emission from the Planetary Nebula BD+30³639. Astrophys. J. Lett. 2000, 545, L57-L59. [CrossRef]

6. Chu, Y.-H.; Guerrero, M.A.; Gruendl, R.A.; Williams, R.M.; Kaler, J.B. Chandra Reveals the X-ray Glint in the Cat's Eye. Astrophys. J. Lett. 2001, 553, L69-L72. [CrossRef]

7. Gruendl, R.A.; Guerrero, M.A.; Chu, Y.-H.; Williams, R.M. XMM-Newton Observations of the Bipolar Planetary Nebulae NGC 2346 and NGC 7026. Astrophys. J. Lett. 2006, 653, 339-344. [CrossRef]

8. Guerrero, M.A.; Chu, Y.-H.; Gruendl, R.A.; Meixner, M. XMM-Newton detection of hot gas in the Eskimo Nebula: Shocked stellar wind or collimated outflows? Astron. Astrophys. 2005, 430, L69-L72. [CrossRef]

9. Guerrero, M.A.; Gruendl, R.A.; Chu, Y.-H. Diffuse X-ray emission from the planetary nebula NGC 7009. Astron. Astrophys. 2002, 387, L1-L5. [CrossRef]

10. Kastner, J.H.; Vrtilek, S.D.; Soker, N. Discovery of Extended X-ray Emission from the Planetary Nebula NGC 7027 by the Chandra X-ray Observatory. Astrophys. J. Lett. 2001, 550, L189-L192. [CrossRef]

11. Kastner, J.H.; Montez, R., Jr.; Balick, B.; De Marco, O. Serendipitous Chandra X-ray Detection of a Hot Bubble within the Planetary Nebula NGC 5315. Astrophys. J. Lett. 2008, 672, 957-961. [CrossRef]

12. Montez, R., Jr.; Kastner, J.H.; De Marco, O.; Soker, N. X-ray Imaging of Planetary Nebulae with Wolf-Rayet-type Central Stars: Detection of the Hot Bubble in NGC 40. Astrophys. J. Lett. 2005, 635, 381-385. [CrossRef]

13. Montez, R., Jr.; Kastner, J.H.; Balick, B.; Frank, A. Serendipitous XMM-Newton Detection of X-ray Emission from the Bipolar Planetary Nebula Hb 5. Astrophys. J. Lett. 2009, 694, 1481-1484. [CrossRef]

14. Ruiz, N.; Chu, Y.H.; Gruendl, R.A.; Guerrero, M.A.; Jacob, R.; Steffen, M. Detection of Diffuse X-ray Emission from Planetary Nebulae with Nebular O VI. Astrophys. J. Lett. 2013, 767, A35. [CrossRef]

15. Kastner, J.H.; Montez, R., Jr.; Balick, B.; Frew, D.J.; Miszalski, B.; Sahai, R.; Blackman, E.; Chu, Y.H.; De Marco, O.; Frank, A.; et al. The Chandra X-ray Survey of Planetary Nebulae (ChanPlaNS): Probing Binarity, Magnetic Fields, and Wind Collisions. Astrophys. J. Lett. 2012, 144, A58. [CrossRef]

16. Freeman, M.; Montez, R., Jr.; Kastner, J.H.; Balick, B.; Frew, D.J.; Jones, D.; Miszalski, B.; Sahai, R.; Blackman, E.; Chu, Y.H.; et al. The Chandra Planetary Nebula Survey (ChanPlaNS). II. X-ray Emission from Compact Planetary Nebulae. Astrophys. J. Lett. 2014, 794, A99. [CrossRef]

17. Montez, R., Jr.; Kastner, J.H.; Balick, B.; Behar, E.; Blackman, E.; Bujarrabal, V.; Chu, Y.H.; Corradi, R.L.; De Marco, O.; Frank, A.; et al. The Chandra Planetary Nebula Survey (ChanPlaNS). III. X-ray Emission from the Central Stars of Planetary Nebulae. Astrophys. J. Lett. 2015, 800, A8. [CrossRef]

18. Sahai, R.; Kastner, J.H.; Frank, A.; Morris, M.; Blackman, E.G. X-ray Emission from the Pre-planetary Nebula Henize 3-1475. Astrophys. J. Lett. 2003, 599, L87-L90. [CrossRef]

19. Steffen, M.; Schönberner, D.; Warmuth, A. The evolution of planetary nebulae. V. The diffuse X-ray emission. Astron. Astrophys. 2008, 489, 173-194. [CrossRef]

20. Toalá, J.A.; Arthur, S.J. Formation and X-ray emission from hot bubbles in planetary nebulae-II. Hot bubble X-ray emission. Mon. Not. R. Astron. Soc. 2016, 463, 4438-4458. [CrossRef] 
21. Yu, Y.S.; Nordon, R.; Kastner, J.H.; Houck, J.; Behar, E.; Soker, N. The X-ray Spectrum of a Planetary Nebula at High Resolution: Chandra Gratings Spectroscopy of BD+30³639. Astrophys. J. Lett. 2009, 690, 440-452. [CrossRef]

22. Guerrero, M.A.; Toalá, J.A.; Chu, Y.-H.; Gruendl, R.A. XMM-Newton RGS observations of the Cat's Eye Nebula. Astron. Astrophys. 2015, 574, A1. [CrossRef]

23. Mellema, G.; Frank, A. Radiation gasdynamics of planetary nebulae-V. Hot bubble and slow wind dynamics. Mon. Not. R. Astron. Soc. 1995, 273, 401-410. [CrossRef]

24. Stute, M.; Sahai, R. X-ray Emission from Planetary Nebulae. I. Spherically Symmetric Numerical Simulations. Astrophys. J. Lett. 2006, 651, 882-897. [CrossRef]

25. Toalá, J.A.; Arthur, S.J. Formation and X-ray emission from hot bubbles in planetary nebulae-I. Hot bubble formation. Mon. Not. R. Astron. Soc. 2014, 443, 3486-3505. [CrossRef]

26. Fang, X.; Zhang, Y.; Kwok, S.; Hsia, C.H.; Chau, W.; Ramos-Larios, G.; Guerrero, M.A. Extended Structures of Planetary Nebulae Detected in $\mathrm{H}_{2}$ Emission. Astrophys. J. Lett. 2018, 859, A92. [CrossRef]

27. Miller Bertolami, M.M. New models for the evolution of post-asymptotic giant branch stars and central stars of planetary nebulae. Astron. Astrophys. 2016, 588, A25. [CrossRef]

28. Soker, N. Backflow in post-asymptotic giant branch stars. Mon. Not. R. Astron. Soc. 2001, 328, 1081-1084. [CrossRef]

29. Chen, Z.; Frank, A.; Blackman, E.G.; Nordhaus, J. The creation of AGB fallback shells. Mon. Not. R. Astron. Soc. 2016, 457, 3219-3224. [CrossRef]

30. Ruiz, N.; Guerrero, M.A.; Chu, Y.-H.; Gruendl, R.A. Physical Structure of the Planetary Nebula NGC 3242 from the Hot Bubble to the Nebular Envelope. Astron. J. 2011, 142, A91. [CrossRef]

31. Chu, Y.-H.; Guerrero, M.A.; Gruendl, R.A.; García-Segura, G.; Wendker, H.J. Hot Gas in the Circumstellar Bubble S308. Astrophys. J. Lett. 2003, 599, 1189-1195. [CrossRef]

32. Toalá, J.A.; Guerrero, M.A.; Chu, Y.H.; Gruendl, R.A.; Arthur, S.J.; Smith, R.C.; Snowden, S.L. X-ray Emission from the Wolf-Rayet Bubble S 308. Astrophys. J. Lett. 2013, 755, A77. [CrossRef]

33. Sahai, R.; Trauger, J.T. Multipolar Bubbles and Jets in Low-Excitation Planetary Nebulae: Toward a New Understanding of the Formation and Shaping of Planetary Nebulae. Astron. J. 1998, 116, 1357-1366. [CrossRef]

34. Dwarkadas, V.V.; Balick, B. The Morphology of Planetary Nebulae: Simulations with Time-evolving Winds. Astrophys. J. Lett. 1998, 497, 267-275. [CrossRef]

35. Huarte-Espinosa, M.; Frank, A.; Balick, B.; Blackman, E.G.; De Marco, O.; Kastner, J.H.; Sahai, R. From bipolar to elliptical: Simulating the morphological evolution of planetary nebulae. Mon. Not. R. Astron. Soc. 2012, 424, 2055-2068. [CrossRef]

36. Lee, C.-F.; Sahai, R. Shaping Proto-Planetary and Young Planetary Nebulae with Collimated Fast Winds. Astrophys. J. Lett. 2003, 586, 319-337. [CrossRef]

37. Akashi, M.; Soker, N. Shaping planetary nebulae by light jets. Mon. Not. R. Astron. Soc. 2008, 391, 1063-1074. [CrossRef]

38. Soker, N. Heat conduction fronts in planetary nebulae. Astron. J. 1994, 107, 276-279. [CrossRef]

39. Zhekov, SA.; Myasnikov, A.V. Colliding Stellar Winds: "Asymmetric" Thermal Conduction. Astrophys. J. Lett. 2000, 543, L53-L56. [CrossRef]

40. Soker, N.; Rahin, R.; Behar, E.; Kastner, J.H. Comparing Shocks in Planetary Nebulae with the Solar Wind Termination Shock. Astrophys. J. Lett. 2010, 725, 1910-1917. [CrossRef]

41. Guerrero, M.A.; Ruiz, N.; Hamann, W.-R.; Chu, Y.H.; Todt, H.; Schönberner, D.; Oskinova, L.; Gruendl, R.A.; Steffen, M.; Blair, W.P.; et al. Rebirth of X-ray Emission from the Born-again Planetary Nebula A 30. Astrophys. J. Lett. 2012, 755, A129. [CrossRef]

42. Toalá, J.A.; Guerrero, M.A.; Todt, H.; Hamann, W.R.; Chu, Y.H.; Gruendl, R.A.; Schönberner, D.; Oskinova, L.M.; Marquez-Lugo, R.A.; Fang, X.; et al. The Born-again Planetary Nebula A 78: An X-ray Twin of A 30. Astrophys. J. Lett. 2015, 799, A67. [CrossRef]

43. López-Martín, L.; Raga, A.C.; Mellema, G.; Henney, W.J.; Cantó, J. Photoevaporating Flows from the Cometary Knots in the Helix Nebula (NGC 7293). Astrophys. J. Lett. 2001, 548, 288-295. [CrossRef] 
44. Mellema, G.; Raga, A.C.; Cantó, J.; Lundqvist, P.; Balick, B.; Steffen, W.; Noriega-Crespo, A. Photo-evaporation of clumps in planetary nebulae. Astron. Astrophys. 1998, 331, 335-346.

45. Nordon, R.; Behar, E.; Soker, N.; Kastner, J.H.; Yu, Y.S. Narrow Radiative Recombination Continua: A Signature of Ions Crossing the Contact Discontinuity of Astrophysical Shocks. Astrophys. J. Lett. 2009, 695, 834-843. [CrossRef]

(C) 2018 by the author. Licensee MDPI, Basel, Switzerland. This article is an open access article distributed under the terms and conditions of the Creative Commons Attribution (CC BY) license (http:/ / creativecommons.org/licenses/by/4.0/). 